\title{
Overexpression of aquaporin-1 aggravates hippocampal damage in mouse traumatic brain injury models
}

\author{
BO QIU ${ }^{1}$, XINGUO LI ${ }^{1}$, XIYANG SUN ${ }^{2}$, YONG WANG ${ }^{1}$, ZHITAO JING $^{1}$, XU ZHANG $^{3}$ and YUNJIE WANG ${ }^{1}$ \\ ${ }^{1}$ Department of Neurosurgery, First Hospital of China Medical University, \\ Shenyang, Liaoning 110001; ${ }^{2}$ Department of Pharmaceutics, School of Pharmacy, \\ Fudan University, Shanghai 201203; ${ }^{3}$ Liaoning Centers for Diseases Control and Prevention, \\ Shenyang, Liaoning 110005, P.R. China
}

Received May 17, 2013; Accepted January 10, 2014

DOI: $10.3892 / \mathrm{mmr} .2014 .1899$

\begin{abstract}
Secondary insult' following primary traumatic brain injury (TBI), including ischemia and edema, may aggravate brain impairments and affect the outcomes. The hippocampus is particularly sensitive to ischemia or edema due to its selective vulnerability, as neural cells of the hippocampus may be more prone to abnormal function or cell death in response to ischemia and edema. Aquaporin-1 (AQP-1) was reported to be associated with cerebral edema; however, the expression and role of AQP-1 in hippocampal edema following TBI have seldom been investigated. In the current study, BALB/c mouse closed craniocerebral injury models were established and the changes of AQP-1 expression in hippocampi of mouse models following TBI were investigated. Neurological function and edema formation of the models were evaluated and the apoptotic hippocampal cells were then stained in situ and detected, followed by determination of AQP-1 expression in the hippocampus using immunohistochemistry and western blot analysis. As a result, the majority of mice in the TBI group were severely injured and hippocampal edema was confirmed. The apoptotic cells increased significantly in the hippocampi of mice in the TBI group compared with those in the sham group $(\mathrm{P}<0.01)$ and the apoptotic rate increased gradually in a time-dependent manner. The expression levels of AQP-1 in the hippocampi of mice were markedly higher in the TBI group than in the sham group $(\mathrm{P}<0.05)$ at various time points and AQP-1 expression levels peaked one day following TBI. These results indicate that upregulation of AQP-1 may participate in edema formation and delayed cell death of the hippocampus following
\end{abstract}

Correspondence to: Professor Bo Qiu, Department of Neurosurgery, First Hospital of China Medical University, 155 North Nanjing Street, Shenyang, Liaoning 110001, P.R. China

E-mail:bo_qiu@msn.com

Key words: secondary injury, vasogenic edema, cytotoxic edema, delayed cell death, BALB/c mice
TBI and may also be a novel therapeutic target to protect the hippocampus from secondary injury following TBI.

\section{Introduction}

Traumatic brain injury (TBI) is a major source of mortality and disability worldwide (1). Usually, the initial mechanical insult (primary injury) is limited and focal. However, these injuries progressively enlarge in extent and severity by mechanisms of secondary injury, the most significant of which are hypoxia, ischemia and edema, which occur within minutes or weeks following the primary injury (1-4). Compared with the primary injury, secondary injuries to the brain are more progressive and may ultimately be the deciding factors in patient recovery (1).

Pathophysiological procedures, including hypoxia, ischemia and edema, are interrelated and mutually improved. The brain consumes almost $20 \%$ of the total oxygen utilized by the body at rest. Due to its high metabolic rate, the brain requires more oxygen and glucose than other organs and is extremely vulnerable to ischemia or hypoxia resulting from decreased cerebral blood flow (CBF), increased intracranial pressure (ICP) and/or swelling and edema (2). Cerebral edema is a fairly common pathophysiological entity among TBI patients and is defined as an excess accumulation of water in the intra- and/or extra-cellular spaces of the brain, which can be induced by vasogenic, cytotoxic or osmotic factors (1,5-7). TBI may result in interstitial (vasogenic) and intracellular (cytotoxic) edema, which contribute to secondary injury (1-3,7). Aside from the forces incurred by the initial trauma, the prolonged compressive forces of cerebral edema may further impair brain function by distorting brain tissues, elevating ICP and reducing $\mathrm{CBF}$, which aggravates ischemia and hypoxia of the brain $(1,2,7)$.

Due to its selective vulnerability, the hippocampus is particularly sensitive to ischemia or edema of secondary injury following TBI (8-12). Damage to the hippocampal region results in neuronal apoptosis and leads to cognitive, memory and learning impairments $(8,10,13,14)$. Therefore, it is crucial to protect hippocampal cells from the insult of ischemia, hypoxia and edema, in order to improve the outcome of TBI. Aquaporins (AQPs) are water channels that provide the major route for water movement across plasma membranes in 
a variety of tissues, including the brain (6,15-20). As a member of the AQPs, AQP-1 has been found to be expressed in astrocytes in cerebral edema associated with TBI, indicating an intimate connection between AQP-1 and cerebral edema (21). In the present study, a series of assays to determine APQ-1 expression in the hippocampal region of mouse models were designed and conducted, and the role of AQP-1 in the hippocampal damage of secondary injury following TBI was evaluated.

\section{Materials and methods}

Animal preparation. All experimental animals were purchased from the Department of Experimental Animal of China Medical University (Shenyang, China). The animals were handled according to the guidelines of the Council for International Organization of Medical Sciences on Animal Experimentation (World Health Organization, Geneva, Switzerland) and the China Medical University. Animal protocols were approved by the Institutional Animal Care and Use Committee (China Medical University). A total of 150 male BALB/c mice (8-10 weeks-old), with an average body weight of 20-25 g, were housed in cages in a light/dark cycle of $12 \mathrm{~h}$. The animals were randomly divided into a control group (sham, $\mathrm{n}=75$ ) and an experimental group (TBI, $\mathrm{n}=75)$; each group contained three subgroups according to sacrifice time $(6,24$ and $72 \mathrm{~h}, \mathrm{n}=25)$ following closed craniocerebral injury (CCI).

Establishment of animal models. Experimental CCI was induced using a modified weight-drop device, as described by Chen et al $(22,23)$. Briefly, following induction of $4-5 \%$ isoflurane anesthesia, a midline longitudinal incision was performed, the skin was retracted and the skull was exposed. The left anterior frontal area was identified as the impact area and a Teflon tipped cone (diameter, $2 \mathrm{~mm}$ ) was placed $1.5 \mathrm{~mm}$ lateral to the midline in the mid-coronal plane. The head was fixed and a 40-g weight was dropped onto the cone from a height of $25 \mathrm{~cm}$, resulting in a focal injury to the left hemisphere. Following trauma, the mice received supporting oxygenation with $95 \% \mathrm{O}_{2}$ for no longer than 2 min and were then returned to their cages. The scalp wound was closed using standard suture material and the wound area was treated with lidocaine cream. Sham controls received anesthesia and skin incision only.

Neurological function score of mouse models. The neurological function of each mouse model was evaluated at various times $(6,24$ and $72 \mathrm{~h}$ ) following CCI using a modified neurological severity score (NSS) as previously described $(22,23)$. Table I shows a set of modified NSS. Neurological function was graded on a scale of 0 to 18 (normal score, 0 ; maximal deficit score, 18). NSS is a composite of motor, sensory, reflex and balance tests. In the severity scores of injury, 1 score point is awarded for the inability to perform the test or for the lack of a tested reflex; thus, the higher the score, the more severe the injury. Thus, a score of 13-18 indicates a 'severe' injury, while a score of 7-12 reflects a 'moderate' injury and $<6$ indicates a 'mild' injury. Initial severity of the trauma was assessed $1 \mathrm{~h}$ following trauma. The parameters of damage for severe injury were established in the present study. A total of 20 mice from each subgroup in the experimental or control groups were randomly selected for each assay (four for edema evaluation, eight for immunochemistry and apoptosis assays and eight for western blot analysis).

Evaluation of edema. As previously described, cerebral edema was measured by detecting the tissue water content in the ipsilateral hippocampus $(22,24)$. Following anesthesia using intraperitoneal administration of $0.8 \%$ pentobarbital (40 mg/kg), a mouse was sacrificed by decapitation and the left hippocampus was rapidly removed. A tissue segment ( $20 \mathrm{mg}$ ) was taken from the left hippocampus and weighed to yield wet weight (WW). Following desiccation oven treatment for $24 \mathrm{~h}$ at $95^{\circ} \mathrm{C}$, the sample was reweighed to yield dry weight (DW). The calculation formula was as follows: Tissue water content $(\%)=[(\mathrm{WW}-\mathrm{DW}) \times 100] / \mathrm{WW}$. Edema was measured at 6,24 and $72 \mathrm{~h}$ following $\mathrm{CCI}$ in four mice at each time point.

Tissue preparation and immunohistochemistry assay. At 6 , 24 and $72 \mathrm{~h}$ following sham surgery or CCI, mice in the sham or TBI groups were anesthetized, sacrificed by decapitation and the whole left hippocampus was rapidly removed and separated on ice. The left hippocampus of each mouse was subject to immunochemistry, apoptosis and western blot analysis.

For immunochemistry and apoptosis detection, the left hippocampus from each mouse was placed in $4 \%$ paraformaldehyde in phosphate-buffered saline [PBS; 0.1 M, (pH 7.6)], containing $0.1 \%$ diethylpyrocarbonate. The tissue was stored in the same fixative solution for $4-6 \mathrm{~h}$ at $4^{\circ} \mathrm{C}$ and then placed in a $30 \%$ phosphate-buffered sucrose at $4^{\circ} \mathrm{C}$ until immersion. The tissue was then paraffin-embedded and $8-\mu \mathrm{m}$ thick coronal sections were created using a rotary microtome (Leica RM2235; Leica Microsystems GmbH, Wetzlar, Germany). Sections were stored at $-80^{\circ} \mathrm{C}$ for later use.

A third series of every tenth section of each left hemisphere was used for detection and quantification of AQP-1 staining. Immunohistochemistry was performed according to the manufacturer's instructions of the Histostain-SP kit (Invitrogen Life Technologies, Carlsbad, CA, USA) and the primary antibody (rabbit anti-mouse AQP-1; Sigma-Aldrich, St. Louis, MO, USA) was diluted at 1:200. AQP-1 binding was visualized using the standard avidin/biotinylated enzyme complex-horseradish peroxidase (HRP) staining procedure, with 3,3'-diaminobenzidine (DAB) as a chromogen. In the control sections, the primary antibody was replaced with normal goat serum. The sections were inspected using an optic microscope (BX40; Olympus, Tokyo, Japan). All experimental groups were included in each immunochemical analysis and were, therefore, processed under the same conditions.

Terminal deoxynucleotidyl transferase (TdT)-mediated dUTP nick-end labeling (TUNEL) staining of hippocampal tissue. Apoptosis of hippocampal cells was detected using an in situ cell death detection kit (Roche Diagnostics, Mannheim, Germany) according to the manufacturer's instructions. Briefly, $8-\mu \mathrm{m}$ paraffin sections underwent conventional deparaffinization and hydration and were then were immersed in $3 \% \mathrm{H}_{2} \mathrm{O}_{2}$ to quench endogenous peroxidase activity, followed by incubation 
Table I. Modified neurological severity score for mice.

Characteristic

Motor tests

Raising rat by the tail

Flexion of forelimb

Flexion of hindlimb

Head moved $>10^{\circ}$ to vertical axis within $30 \mathrm{sec}$

Placing rat on the floor

Normal walk

Inability to walk straight

Circling toward the paretic side

Fall down to the paretic side

Sensory tests

Placing test (visual and tactile test)

Proprioceptive test (deep sensation, pushing the paw against the table edge to stimulate limb muscles)

Beam balance tests

Balances with steady posture

Grasps side of beam

Hugs the beam and one limb falls down from the beam

Hugs the beam and two limbs fall down from the beam or spins on beam ( $>60 \mathrm{sec})$

Attempts to balance on the beam but falls off ( $>40 \mathrm{sec})$

Attempts to balance on the beam but falls off $(<20 \mathrm{sec})$

Falls off: No attempt to balance or hang on to the beam $(<20 \mathrm{sec})$

Reflexes absent and abnormal movements

Pinna reflex (head shake when touching the auditory meatus)

Corneal reflex (eye blink when lightly touching the cornea with cotton)

Startle reflex (motor response to a brief noise from snapping a clipboard paper)

Seizures, myoclonus, myodystony

Maximum points, 18 . One point is awarded for the inability to perform the tasks or for the lack of a tested reflex; 13-18 indicates severe injury; 7-12, moderate injury; and 1-6, mild injury.

at room temperature for $15 \mathrm{~min}$ with proteinase $\mathrm{K}[20 \mu \mathrm{g} / \mathrm{ml}$ in Tris/ $\mathrm{HCl}$ (pH 7.4-8.0); Promega Corporation, Madison, WI, USA]. Thereafter, sections were incubated with $50 \mu 1$ TUNEL reaction mixture (TdT and labeled nucleotide mixture) for $1 \mathrm{~h}$ in $37^{\circ} \mathrm{C}$. The sections were rinsed in PBS, wiped dry, incubated with $50 \mu \mathrm{l}$ converter-peroxidase for $30 \mathrm{~min}$ at $37^{\circ} \mathrm{C}$, washed again and further incubated with $50 \mu \mathrm{l}$ DAB substrate solution (Roche Diagnostics) at room temperature for $10 \mathrm{~min}$. The sections were subsequently dehydrated, cleared and mounted. Cells containing fragmented nuclear chromatin exhibited a brown nuclear stain. As negative controls, sections were processed without TdT buffer. Apoptotic cells were examined with an optic microscope (BX40).

Western blot analysis. For western blot analysis, the left hippocampus removed from each mouse was used for sodium dodecyl sulfate-polyacrylamide gel electrophoresis (SDS-PAGE) and western blot analysis, as described previously (25). Equal quantities of tissue lysates were resolved by SDS-PAGE, transferred onto polyvinylidene fluoride membranes (Roche Diagnostics) using electroblotting, probed with specific primary antibodies followed by secondary antibody conjugation and then analyzed. The primary antibodies were monoclonal rabbit anti-mouse AQP-1 (1:500; Sigma-Aldrich) and monoclonal anti- $\beta$-actin antibody (1:500; Santa Cruz Biotechnology Inc., Santa Cruz, CA, USA) and were detected using HRP-conjugated goat anti-mouse $\operatorname{IgG}$ (1:500; Sigma-Aldrich) in blocking solution. The non-glycosylated $(28 \mathrm{kDa})$ band was used for analysis of changes in AQP-1 expression in all experiments. Immunoreactive protein bands were visualized with enhanced chemiluminescence reagent (ECL-PLUS; Amersham Biosciences, Piscataway, NJ, USA), scanned with a FujiFilm LAS300 Intelligent Dark Box scanner (Fujifilm, Tokyo, Japan) and quantified for pixel density by the optical density (OD) method using Image J software (version 1.46; http://rsb.info.nih.gov/ij/).

Statistical analysis. All analyses were performed in triplicate and the representative results are presented as the mean \pm standard deviation (SD). The data were analyzed using SPSS for Windows software (version 18.0; SPSS, Inc., 
A

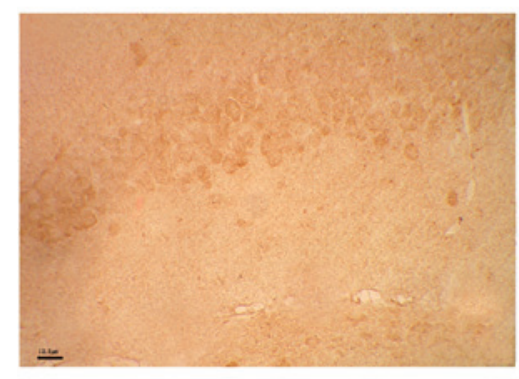

B

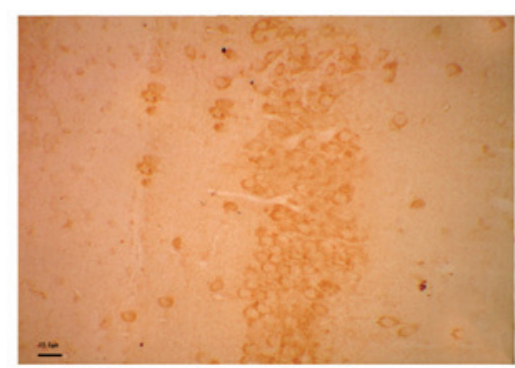

AQP-1 expression in hippocampus

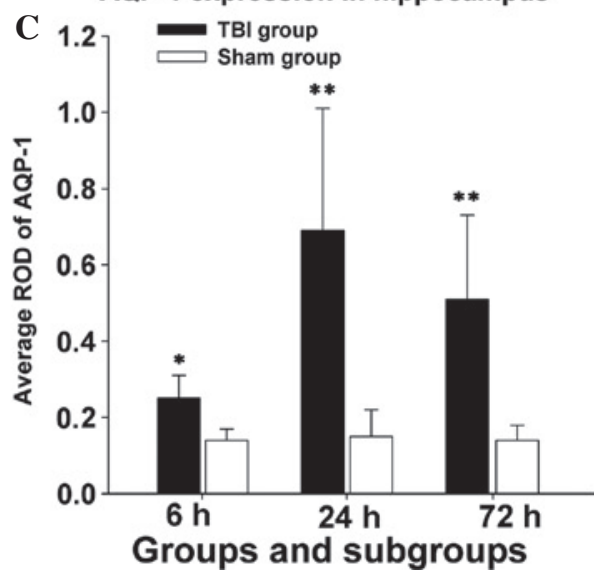

Figure 1. AQP-1 expression in hippocampi was detected using an immunochemistry assay. (A) AQP-1 was expressed at uniformly low levels in the hippocampi of the sham group at various times (72 h; scale bar, $12.5 \mu \mathrm{m}$; magnification, $\mathrm{x} 200)$. (B) Expression levels of AQP-1 in hippocampi of the TBI group increased significantly following closed craniocerebral injury (72 h; scale bar, $12.5 \mu \mathrm{m}$; magnification, x200). (C) Histogram of AQP-1 expression levels in the experimental and control groups at various times. ${ }^{*} \mathrm{P}<0.05$ and ${ }^{* *} \mathrm{P}<0.01$ vs. sham. AQP-1, aquaporin-1; TBI, traumatic brain injury.

Chicago, IL, USA). Comparisons between groups were made with a one-way analysis of variance and a paired t-test. $\mathrm{P}<0.05$ or $\mathrm{P}<0.01$ were considered to indicate a statistically significant difference.

\section{Results}

Outcomes of mouse models. In a preliminary experiment, the weight and distance selected for the object in the weight-drop device were repeatedly tested to guarantee that the average NSS score for the majority of mouse models was confined to the range of severe injury (score, 13-18). The clinical statuses of the injured mice were evaluated by the modified NSS at 6 , 24 and $72 \mathrm{~h}$ following CCI. The average NSS was found to decrease from $15.3 \pm 0.9$ at $1 \mathrm{~h}$, to $14.2 \pm 1.1$ at $6 \mathrm{~h}, 11.2 \pm 0.6$ at $24 \mathrm{~h}$ and $10.6 \pm 0.8$ at $72 \mathrm{~h}$ following CCI. In the control group, mice that received sham surgery were able to perform all tasks and obtained a score of 0 in the NSS.

Edema formation. The experimental CCI resembled cerebral edema following TBI. In the control group, the average cerebral water content in the left hippocampi at various times showed no marked fluctuation $(77.23 \pm 0.46 \%, 77.51 \pm 0.35 \%$ and $77.48 \pm 0.51 \%$ at 6,24 and $72 \mathrm{~h}$, respectively). In the experimental group, edema was detected $6 \mathrm{~h}$ following CCI in the left hippocampus $(79.44 \pm 0.36 \% ; \mathrm{P}<0.05$, vs. sham), peaked at $24 \mathrm{~h}(81.28 \pm 0.20 \%$; $\mathrm{P}<0.01$, vs. sham) and maintained a high level at $72 \mathrm{~h}(80.73 \pm 0.34 \%$; $\mathrm{P}<0.01$, vs. sham). These results demonstrated the existence of acute hippocampal edema following experimental CCI.

Outcomes of immunohistochemistry. Immunohistochemical staining for AQP-1 was evaluated qualitatively in at least ten serial sections of each hippocampus from a subgroup of experimental or sham groups and results of ten separate measurements were expressed as the mean \pm SD. The intensity of the immunohistochemical reaction was expressed as relative OD (ROD) of DAB brown reaction product and calculated using Image-Pro Plus for Windows (Version 6.0; Media
Cybernetics, Bethesda, MD, USA) using the following formula where GL is the gray level for the stained area (specimen, S) and unstained area (background, BG) and blank (B) is the gray level measured following removal of the slide from the light path: $\mathrm{ROD}=\mathrm{OD}_{\mathrm{S}} / \mathrm{OD}_{\mathrm{BG}}=\log \left(\mathrm{GL}_{\mathrm{B}} / \mathrm{GL}_{\mathrm{S}}\right) / \log \left(\mathrm{GL}_{\mathrm{B}} / \mathrm{GL}_{\mathrm{BG}}\right)$.

In the immunohistochemistry assay, AQP-1 expression levels in mice hippocampi significantly increased $6 \mathrm{~h}$ following CCI compared with those in the sham group at the same time (average, $0.25 \pm 0.06$ vs. $0.14 \pm 0.03$; $\mathrm{P}<0.05$ ). AQP-1 expression in the experimental group reached peak levels at $24 \mathrm{~h}$ following CCI $(0.69 \pm 0.32$ vs. $0.15 \pm 0.07 ; \mathrm{P}<0.01)$. Following 72 h CCI, AQP-1 expression marginally decreased, but remained markedly higher than that in the sham group at the same time point $(0.51 \pm 0.22$ vs. $0.14 \pm 0.04 ; \mathrm{P}<0.01)$. The difference in mean ROD of AQP-1 protein levels in mice hippocampi between the experimental or sham group was statistically significant at various times $(6,24$ and $72 \mathrm{~h})$, as shown in Fig. 1.

Apoptosis detection in the hippocampus following TBI. In the control group, the average apoptotic rate of hippocampal cells was relatively constant and low at various times $(8.61 \pm 8.25$, $7.55 \pm 9.54$ and $10.17 \pm 6.08 \%$ at 6,24 and $72 \mathrm{~h}$ following sham surgery, respectively). In the experimental group, however, the average apoptotic rate increased with prolonged post-injury interval. A marked increase in TUNEL-positive cells was observed $6 \mathrm{~h}$ following injury $(44.26 \pm 15.18 \%)$, which was significantly higher than its counterpart in the sham group $(\mathrm{P}<0.01)$. The average apoptotic rate of hippocampal cells in the experimental group continued to rise at $24 \mathrm{~h}$ following injury $(53.35 \pm 22.67 \%)$ and reached $61.62 \pm 26.55 \%$ at $72 \mathrm{~h}$ following injury, which were significantly higher values than those in corresponding subgroups of the control group $(\mathrm{P}<0.01$ for the two comparisons), as shown in Fig. 2.

Quantitation of AQP-1 protein expression. The present study investigated whether AQP-1 expression in BALB/c mice was altered following experimental TBI. Western blot analysis showed one band at $28 \mathrm{kDa}$ corresponding 
A

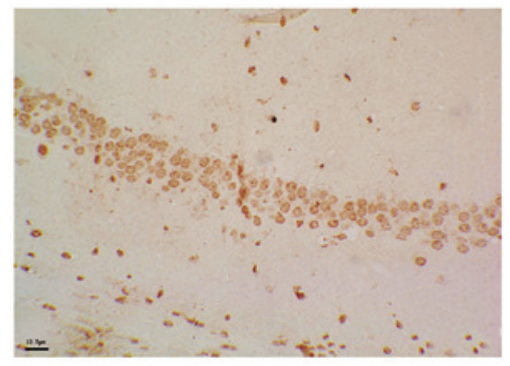

B

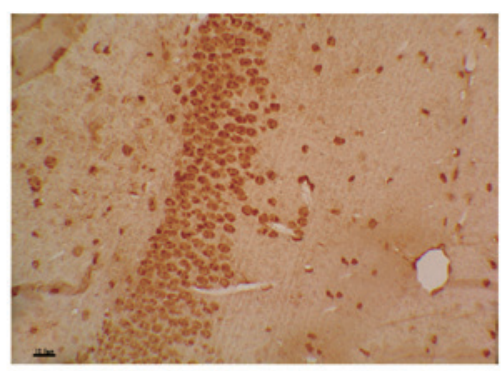

C In situ apoptosis detection by TUNEL

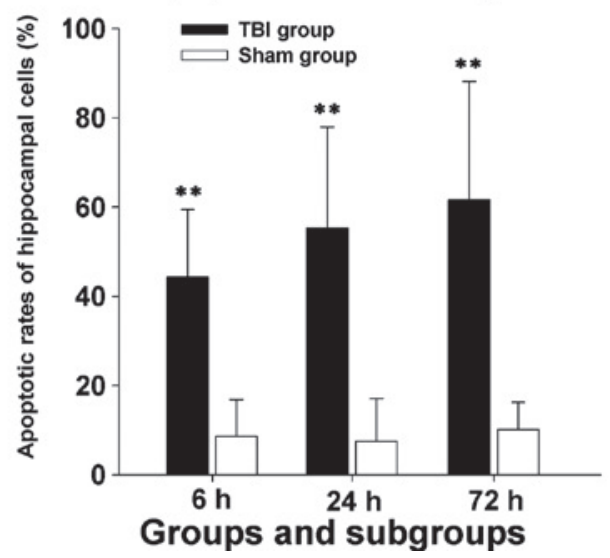

Figure 2. Apoptotic rates of hippocampal cells in all groups by TUNEL. (A) Average apoptotic rate of hippocampal cells was constantly low following sham surgery ( $72 \mathrm{~h}$; scale bar, $12.5 \mu \mathrm{m}$; magnification, $\mathrm{x} 200)$. (B) In the TBI group, average apoptotic rate of hippocampal cells increased continuously following closed craniocerebral injury (72 h; scale bar, $12.5 \mu \mathrm{m}$; magnification, x200). (C) Histogram of average apoptotic rate of hippocampal cells in experimental and control groups at various times. ${ }^{* *} \mathrm{P}<0.01$ vs. the sham group. TBI, traumatic brain injury; TUNEL, terminal deoxynucleotidyl transferase-mediated dUTP nick-end labeling.

$\mathbf{A}$
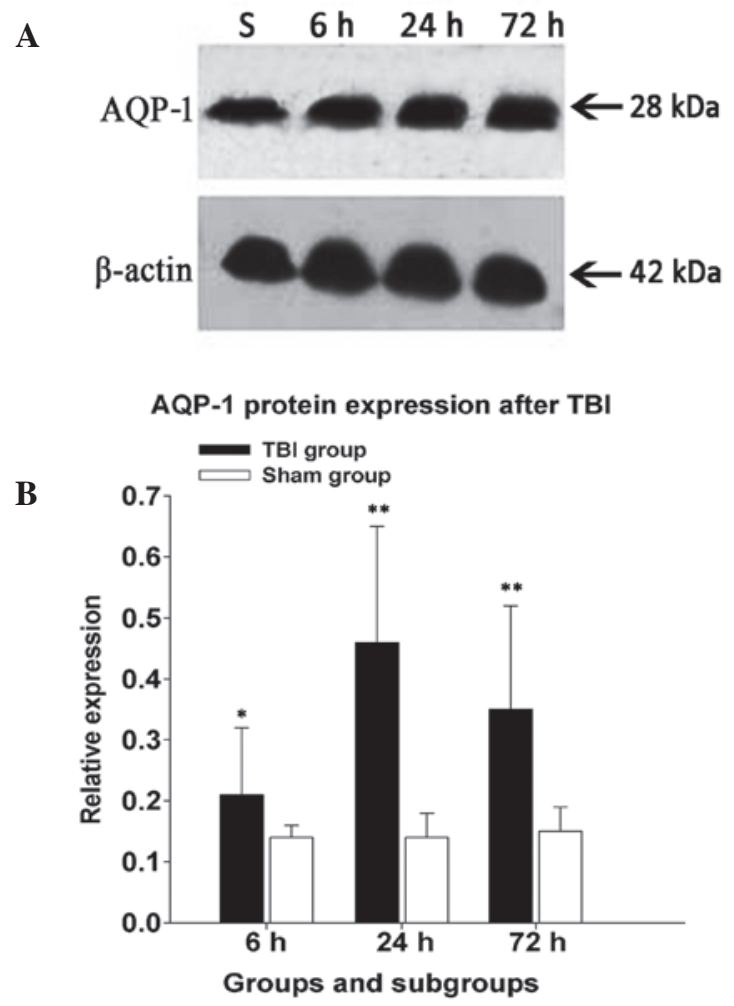

Figure 3. Western blot analysis of AQP-1. (A) Non-glycosylated (28 kDa) AQP-1 expression levels in the hippocampus increased $6 \mathrm{~h}$ following TBI, peaked at $24 \mathrm{~h}$ and decreased when detected at $72 \mathrm{~h}$ following injury. (B) Paired t-tests confirmed that the variations in AQP-1 expression between subgroups from the experimental and control groups at the same time points were statistically significant. ${ }^{*} \mathrm{P}<0.05$ and ${ }^{* *} \mathrm{P}<0.01$ vs. sham. $\mathrm{S}$, sham group; 6,24 and 72 h, TBI group at various time points. AQP-1, aquaporin-1; TBI, traumatic brain injury.

to non-glycosylated AQP-1. There were significant differences in the expression of AQP-1 between subgroups of the experimental and control groups at various times (Fig. 3). The average AQP-1 expression level in the hippocampi of the experimental group increased to $0.21 \pm 0.11$ at $6 \mathrm{~h}$, peaked
$(0.46 \pm 0.19)$ at $24 \mathrm{~h}$ and marginally decreased to $0.35 \pm 0.17$ at $72 \mathrm{~h}$ following experimental TBI. Compared with those in the control group at various times $(0.14 \pm 0.02,0.14 \pm 0.04$ and $0.15 \pm 0.04$, at 6,24 and $72 \mathrm{~h}$, respectively), the expression of AQP-1 protein was markedly upregulated following TBI, which was in accordance with the results of immunohistochemistry assay.

\section{Discussion}

Secondary insult of TBI includes complicated biomolecular and physiological changes that destroy surviving cells of the primary injury and cause delayed cell death in surrounding or distant regions $(2,26)$. Currently, post-injury hypoxia, ischemia and edema have attracted increasing attention in TBI research $(1-4,7)$. The contribution of cerebral edema to brain swelling in cases of TBI remains a critical problem for which there is currently no effective clinical treatment (7). Notably, a previous study has demonstrated that the degree of brain swelling assessed on the first computed tomography scan, obtained soon after injury, correlates highly with mortality rate (e.g., compressed or obliterated mesencephalic cisterns vs. normal cisterns, $\mathrm{P}<0.0002)(27)$, indicating that therapy must be commenced as soon as possible to avoid neurological deterioration or mortality (7). According to present hypotheses, vasogenic edema may result from a failure of the blood-brain barrier, while cytotoxic edema is the result of a biomolecular injury and inflammatory cascade that causes loss of ionic gradients, membrane dysfunction and cellular swelling $(7,12,28)$. As a result of traumatic impact or the subsequent attack of free radicals, permeability of the blood-brain barrier or reflex vasodilation of vessels increases and allows the plasma components of blood to gain entrance to the extracellular space, indicating an 'original' vasogenic edema $(1,2,7,13,14,29)$. Additionally, ischemia and hypoxia, in the process of secondary injury, may deplete cell energy stores and disable the sodium-potassium ATPase. This reduces calcium exchange and, thereby, results in cytotoxic 
cerebral edema (1,3). Moreover, cellular swelling may, in turn, aggravate the cellular dysfunction and cytotoxic edema $(1,3)$. Furthermore, previous studies of TBI have indicated that the predominant type of edema in these injuries is cellular rather than vasogenic $(30,31)$. Therefore, the mechanisms of water transport across the plasma membrane of brain cells are of importance.

The identification of AQPs was acclaimed enthusiastically, given that they may provide a mechanism for massive water movement across the cell membrane (6,7,16-20). Despite a common molecular structure, mammalian AQPs have been subdivided into three functional groups according to permeability characteristics: the AQPs (AQP-0, $-1,-2,-4,-5$ and -6), which are permeable to water; the aquaglyceroporins (AQP-3, -7 and -8$)$, which are permeable to water and small nonpolar solutes; and the neutral solute channels (AQP-9), which are permeable to water and specific neutral solutes $(7,18,32)$. At present, AQPs are considered to contribute to cerebral edema secondary to TBI, cerebral ischemia or hypoxia $(6,16,18,33)$. Although AQP-4 and -9 have been proven to be important in cerebral edema following TBI or cerebral ischemia or hypoxia $(6,7,16,18,33,34)$, AQP-1, as the first AQP to be identified, was seldom investigated in cerebral edema following TBI, despite there being evidence that AQP-1 may participate in acute lung edema (35). In addition, the hippocampus is an important organ that is extremely sensitive to ischemia, hypoxia and edema, which may lead to hippocampal cell apoptosis and subsequent cognitive, memory and learning impairments or epilepsy, thereby profoundly affecting prognosis (8-14). To the best of our knowledge, expression of AQP-1 in the hippocampus following TBI has not yet been investigated. However, studies have reported that upregulated AQP-1 expression following acute hyponatremia correlates with necrotic cell mortality of the hippocampal CA1 region, increasing water transport across the blood-cerebrospinal fluid (CSF) barrier (19) or in vacuolized astrocytes in the stratum radiatum of the CA1 region in an animal epilepsy model (15). Thus, in the present study, a series of assays was performed to develop mouse TBI models and detect AQP-1 expression in the hippocampus following trauma and, subsequently, to evaluate its possible effects in hippocampal injuries correlated with TBI.

In this study, the mouse models were well established and mimicked clinical manifestations of TBI. Post-injury NSS confirmed that the majority of mice in the TBI group were severely injured and attained the experimental requirements. Acute hippocampal edema was also reproduced and confirmed by evaluation of hippocampal tissue water content. In situ apoptosis detection of hippocampal cells demonstrated a progressive increase of apoptosis in the TBI group over time, whereas the average apoptotic rate of hippocampal cells in the sham group was maintained at a low and stable level. The differences were statistically significantly $(\mathrm{P}<0.01$ for all comparisons), indicating an activation of hippocampal apoptosis following experimental TBI, in which several pathophysiological processes may be involved. The average apoptotic rate increased gradually in a time-dependent manner, implying secondary or delayed cell mortality or damage, resulting from physiological and biochemical changes induced by the primary insult (26).
AQP-1 was formerly found to be expressed on epithelial cells of the choroid plexus, with a role in CSF production; however, more recently AQP-1 has been found to be expressed in microvascular endothelial cells or astrocytes, particularly in a number of pathological states, including brain injury, subarachnoid hemorrhage or brain tumors, leading to edema formation $(16,21,26,36-38)$. We hypothesize that AQP-1 is involved in TBI-associated cerebral edema and this protein may be upregulated in response to a TBI insult. Immunohistochemistry and western blot analysis substantiated the upregulation of AQP-1 expression following TBI, and the acute hippocampal edema was formed and evolved. Results of the two assays were consistent, demonstrating enhanced AQP-1 expression levels at $6 \mathrm{~h}$, maximum expression at $24 \mathrm{~h}$ and mildly decreased (although remaining relatively high) expression at $72 \mathrm{~h}$ following injury. These observations imply the involvement of AQP-1 in post-TBI pathophysiological and biochemical changes in the hippocampus. Moreover, Kim et al (15) reported that AQP-1 overexpression correlates with vacuolization of hippocampal CA1 astrocytes, which may be responsible for subsequent apoptosis. Jablonski et al (39) reported that AQP-1-mediated water loss is important for the apoptotic volume decrease and downstream apoptotic events and that the water permeability of the plasma membrane may control the rate of apoptosis. Therefore, in light of concurrent hippocampal edema and apoptosis exhibiting a similar trend of development, it may be inferred that AQP-1 participates in edema formation and delayed cell mortality of the hippocampus.

Currently, several studies have identified that AQP-1 participates in the mediation of vasogenic edema $(21,40)$; furthermore, there is specific evidence to indicate that AQP-1 channels facilitate membrane $\mathrm{CO}_{2}$ permeability, contributing to intracellular acidification $(17,41)$. Besides vasogenic edema, AQP-1-induced intracellular acidification is likely to contribute to cytotoxic edema of the hippocampus in that intracellular acidosis leads to ion transport dysfunction of the cell membrane and subsequent cell swelling (17,42-45). Additionally, vasogenic and cytotoxic edema may exacerbate cell metabolic disorders and acidosis and, in turn, aggravate cerebral edema $(7,12,28)$. Selective vulnerability disposes the hippocampus to a high susceptibility to these pathophysiological attacks. As a result, the post-TBI apoptotic cells in the hippocampus increased in a time-dependent manner in the current study.

In conclusion, AQP-1 expression is upregulated in the hippocampus of a mouse model following experimental TBI and may exhibit differential roles in various responses, including post-TBI hippocampal edema and apoptosis. Therefore, observations of the present study indicate that AQP-1 may be a novel therapeutic target to protect the hippocampus from secondary injury following TBI.

\section{Acknowledgements}

This study was supported by grants from the Chinese National Natural Science Foundation of Youth Science Foundation (grant nos. 81000565 and 81101917), the China Postdoctoral Science Foundation (grant no. 2012M520831) and the Liaoning Provincial Natural Science Foundation (grant nos. 201102095 and 2013021075). 


\section{References}

1. Greve MW and Zink BJ: Pathophysiology of traumatic brain injury. Mt Sinai J Med 76: 97-104, 2009.

2. Borgens RB and Liu-Snyder P: Understanding secondary injury. Q Rev Biol 87: 89-127, 2012.

3. Werner $\mathrm{C}$ and Engelhard K: Pathophysiology of traumatic brain injury. Br J Anaesth 99: 4-9, 2007.

4. Padayachy LC, Rohlwink U, Zwane E, Fieggen G, Peter JC and Figaji AA: The frequency of cerebral ischemia/hypoxia in pediatric severe traumatic brain injury. Childs Nerv Syst 28: 1911-1918, 2012.

5. Walcott BP, Kahle KT and Simard JM: Novel treatment targets for cerebral edema. Neurotherapeutics 9: 65-72, 2012.

6. Adeva MM, Souto G, Donapetry C, Portals M, Rodriguez A and Lamas D: Brain edema in diseases of different etiology. Neurochem Int 61: 166-174, 2012.

7. Marmarou A: A review of progress in understanding the pathophysiology and treatment of brain edema. Neurosurg Focus 22: E1, 2007.

8. Schmidt-Kastner R and Freund TF: Selective vulnerability of the hippocampus in brain ischemia. Neuroscience 40: 599-636, 1991

9. Royo NC, Conte V, Saatman KE, et al: Hippocampal vulnerability following traumatic brain injury: a potential role for neurotrophin-4/5 in pyramidal cell neuroprotection. Eur J Neurosci 23: 1089-1102, 2006.

10. Deng $\mathrm{P}$ and $\mathrm{Xu} \mathrm{ZC}$ : Contribution of Ih to neuronal damage in the hippocampus after traumatic brain injury in rats J Neurotrauma 28: 1173-1183, 2011.

11. Nawashiro H, Shima K and Chigasaki H: Selective vulnerability of hippocampal CA3 neurons to hypoxia after mild concussion in the rat. Neurol Res 17: 455-460, 1995.

12. Kawamata T, Katayama Y, Tsuji N and Nishimoto H: Metabolic derangements in interstitial brain edema with preserved blood flow: selective vulnerability of the hippocampal CA3 region in rat hydrocephalus. Acta Neurochir Suppl 86: 545-547, 2003.

13. Deng W, Aimone JB and Gage FH: New neurons and new memories: how does adult hippocampal neurogenesis affect learning and memory? Nat Rev Neurosci 11: 339-350, 2010.

14. Eichenbaum H: Hippocampus: cognitive processes and neural representations that underlie declarative memory. Neuron 44: 109-120, 2004.

15. Kim JE, Ryu HJ, Yeo SI, et al: Differential expressions of aquaporin subtypes in astroglia in the hippocampus of chronic epileptic rats. Neuroscience 163: 781-789, 2009.

16. Zelenina M: Regulation of brain aquaporins. Neurochem Int 57: 468-488, 2010

17. Tran ND, Kim S, Vincent HK, et al: Aquaporin-1-mediated cerebra edema following traumatic brain injury: effects of acidosis and corticosteroid administration. J Neurosurg 112: 1095-1104, 2010.

18. Badaut J, Lasbennes F, Magistretti PJ and Regli L: Aquaporins in brain: distribution, physiology, and pathophysiology. J Cereb Blood Flow Metab 22: 367-378, 2002.

19. Kim J and Jung Y: Increased aquaporin- 1 and $\mathrm{Na}^{+}-\mathrm{K}^{+}-2 \mathrm{Cl}^{-}$cotransporter 1 expression in choroid plexus leads to blood-cerebrospinal fluid barrier disruption and necrosis of hippocampal CA1 cells in acute rat models of hyponatremia. J Neurosci Res 90: 1437-1444, 2012.

20. Ameli PA, Madan M, Chigurupati S, Yu A, Chan SL and Pattisapu JV: Effect of acetazolamide on aquaporin-1 and fluid flow in cultured choroid plexus. Acta Neurochir Suppl 113: 59-64, 2012

21. Suzuki R, Okuda M, Asai J, et al: Astrocytes co-express aquaporin-1, -4, and vascular endothelial growth factor in brain edema tissue associated with brain contusion. Acta Neurochir Suppl 96: 398-401, 2006.

22. Chen Y, Constantini S, Trembovler V, Weinstock $M$ and Shohami E: An experimental model of closed head injury in mice: pathophysiology, histopathology, and cognitive deficits. J Neurotrauma 13: 557-568, 1996.

23. Albert-Weißenberger C, Várrallyay C, Raslan F, Kleinschnitz C and Sirén AL: An experimental protocol for mimicking pathomechanisms of traumatic brain injury in mice. Exp Trans Stroke Med 4: 1, 2012.
24. Shapira Y, Shohami E, Sidi A, Soffer D, Freeman S and Cotev S: Experimental closed head injury in rats: mechanical, pathophysiologic, and neurologic properties. Crit Care Med 16: 258-265, 1988.

25. Qiu B, Sun X, Zhang D, Wang Y, Tao J and Ou S: TRAIL and paclitaxel synergize to kill U87 cells and U87-derived stem-like cells in vitro. Int J Mol Sci 13: 9142-9156, 2012.

26. Stoica BA and Faden AI: Cell death mechanisms and modulation in traumatic brain injury. Neurotherapeutics 7: 3-12, 2010.

27. Eisenberg HM, Gary HE Jr, Aldrich EF, et al: Initial CT findings in 753 patients with severe head injury. A report from the NIH Traumatic Coma Data Bank. J Neurosurg 73: 688-698, 1990.

28. Donkin JJ and Vink R: Mechanisms of cerebral edema in traumatic brain injury: therapeutic developments. Curr Opin Neurol 23: 293-299, 2010.

29. Barzó P, Marmarou A, Fatouros P, Hayasaki K and Corwin F: Contribution of vasogenic and cellular edema to traumatic brain swelling measured by diffusion-weighted imaging. J Neurosurg 87: 900-907, 1997.

30. Ito J, Marmarou A, Barzó P, Fatouros P and Corwin F: Characterization of edema by diffusion-weighted imaging in experimental traumatic brain injury. J Neurosurg 84: 97-103, 1996.

31. Unterberg AW, Stover J, Kress B and Kiening KL: Edema and brain trauma. Neuroscience 129: 1021-1029, 2004.

32. Verkman AS, Yang B, Song Y, Manley GT and Ma T: Role of water channels in fluid transport studied by phenotype analysis of aquaporin knockout mice. Exp Physiol 85 Spec No: 233S-241S, 2000.

33. Badaut J, Hirt L, Granziera C, Bogousslavsky J, Magistretti PJ and Regli L: Astrocyte-specific expression of aquaporin-9 in mouse brain is increased after transient focal cerebral ischemia. J Cereb Blood Flow Metab 21: 477-482, 2001.

34. Badaut J: Aquaglyceroporin 9 in brain pathologies. Neuroscience 168: 1047-1057, 2010.

35. Wang Q, Ishikawa T, Michiue T, Zhu BL, Guan DW and Maeda H: Molecular pathology of pulmonary edema after injury in forensic autopsy cases. Int J Legal Med 126: 875-882, 2012.

36. Kobayashi H, Minami S, Itoh S, et al: Aquaporin subtypes in rat cerebral microvessels. Neurosci Lett 297: 163-166, 2001.

37. Badaut J, Brunet JF, Grollimund L, et al: Aquaporin 1 and aquaporin 4 expression in human brain after subarachnoid hemorrhage and in peritumoral tissue. Acta Neurochir Suppl 86: 495-498, 2003

38. Satoh J, Tabunoki H, Yamamura T, Arima K and Konno H: Human astrocytes express aquaporin-1 and aquaporin-4 in vitro and in vivo. Neuropathology 27: 245-256, 2007.

39. Jablonski EM, Webb AN, McConnell NA, Riley MC and Hughes FM Jr: Plasma membrane aquaporin activity can affect the rate of apoptosis but is inhibited after apoptotic volume decrease. Am J Physiol Cell Physiol 286: C975-985, 2004.

40. Kim J and Jung Y: Different expressions of AQP1, AQP4, eNOS, and VEGF proteins in ischemic versus non-ischemic cerebropathy in rats: potential roles of AQP1 and eNOS in hydrocephalic and vasogenic edema formation. Anat Cell Biol 44: 295-303, 2011.

41. Blank ME and Ehmke $\mathrm{H}$ : Aquaporin-1 and $\mathrm{HCO}_{3}(-)-\mathrm{Cl}^{-}$transporter-mediated transport of $\mathrm{CO}_{2}$ across the human erythrocyte membrane. J Physiol 550: 419-429, 2003.

42. Staub F, Winkler A, Haberstok J, et al: Swelling, intracellular acidosis, and damage of glial cells. Acta Neurochir Suppl 66: 56-62, 1996.

43. Ringel F, Plesnila N, Chang RC, Peters J, Staub F and Baethmann A: Role of calcium ions in acidosis-induced glial swelling. Acta Neurochir Suppl 70: 144-147, 1997.

44. Plesnila N, Haberstok J, Peters J, Kolbl I, Baethmann A and Staub F: Effect of lactacidosis on cell volume and intracellular pH of astrocytes. J Neurotrauma 16: 831-841, 1999.

45. Ringel F, Chang RC, Staub F, Baethmann A and Plesnila N: Contribution of anion transporters to the acidosis-induced swelling and intracellular acidification of glial cells. J Neurochem 75: 125-132, 2000. 